\title{
CHARACTERIZING CIRCULAR-ARC GRAPHS ${ }^{1}$
}

\author{
BY ALAN TUCKER
}

Communicated by V. L. Klee, April 16, 1970

Given a finite family $S$ of nonempty sets, the intersection graph $G=G(S)$ has vertices associated with the sets of $S$ and two distinct vertices of $G$ are adjacent if and only if the corresponding sets of $S$ intersect ( $S$ is called an intersection model for $G$ ). If $S$ is a family of arcs on a circle, $G$ is called a circular-arc graph. See the example in Figure 1. If, in addition, no arc of $S$ contains another arc, $G$ is called a proper circular-arc graph (the graph in Figure 1 is not a proper cir-

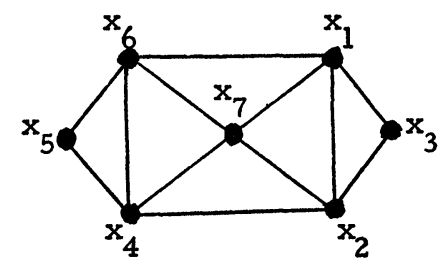

Figure 1a: $G$

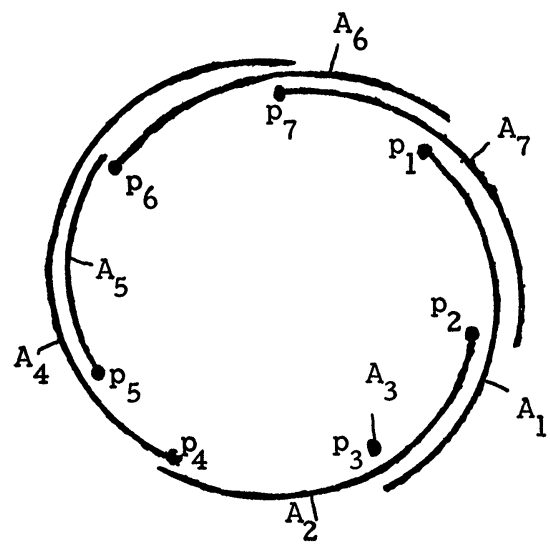

Figure 1b: Circular-arc Model of $G$

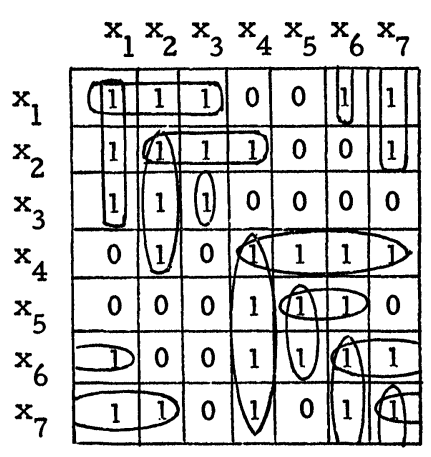

Figure 1c: $M^{*}(G)$ with $U_{i}$ 's and $V_{i}$ 's circled

FIGURE 1

AMS 1969 subject classifications. Primary 0525, 0540; Secondary 1548, 9210.

Key words and phrases. Interval graph, proper circular-arc graph, intersection graph, circular 1's property, consecutive 1's property.

1 Sponsored by the Mathematics Research Center, United States Army, Madison, Wisconsin, under Contract No. DA-31-124-ARO-D-462. 
cular-arc graph). Interval graphs and proper interval graphs are analogously defined. Fulkerson and Gross [3] characterized interval graphs as graphs whose dominant clique-vertex incidence matrix has the consecutive 1's property for columns, that is, the rows of this matrix can be permuted so that the 1 's appear consecutively in each column. For other characterizations of interval graphs, see Gilmore and Hoffman [4] and Lekkerkerker and Boland [8]. The study of interval graphs was motivated by the central role they played in some work by Benzer [1] concerning the molecular substructure of genes. More recently, interval graphs have been applied to problems in archeology [6] and ecology [2]. Proper interval graphs have been characterized by Roberts [9], [10] with a structure theorem and as graphs whose augmented adjacency matrix (defined below) has the consecutive 1's property for columns. He showed that they were equivalent to the indifference graphs of mathematical psychology.

The problem of characterizing circular-arc graphs was posed in [5] and recently discussed in some detail by Klee [7]. Such graphs may be applicable in testing for circular arrangements of genetic molecules (see [7]). In this note, we present characterizations of circulararc and proper circular-arc graphs in terms of their augmented adjacency matrices.

We shall deal throughout with graphs in which the set of vertices is finite and the adjacency relation is symmetric and irreflexive (no loops). Associated with a graph $G$ is an adjacency matrix $M(G)$ defined with entry $(i, j)=1$ if vertices $x_{i}$ and $x_{j}$ are adjacent, and $=0$ otherwise. We define $M^{*}(G)$, the augmented adjacency matrix, to be $M^{*}(G)=M(G)+I$, i.e., $M(G)$ with 1 's added on the main diagonal. A $(0,1)$-matrix is said to have the circular 1's property for columns if the rows of $M$ can be permuted so that the 1's in each column are circular, that is, appear in a circularly consecutive fashion (think of the matrix as wrapped around a cylinder).

Theorem 1. Let $M_{1}$ be a $(0,1)$-matrix. Form the matrix $M_{2}$ from $M_{1}$ by complementing (interchanging 0's and 1's) those columns with a 1 in the first row of $M_{1}$. Then $M_{1}$ has the circular 1's property for columns if and only if $M_{2}$ has the consecutive 1's property for columns.

Fulkerson and Gross [3] have described an efficient algorithm to test whether a $(0,1)$-matrix has the consecutive 1 's property for columns and to obtain a desired row permutation when one exists. Using Theorem 1, their algorithm now solves the corresponding problem for circular 1's.

Let $M$ be a symmetric $(0,1)$-matrix with 1 's on the main diagonal. 
Let $U_{i}$ be the circular set of 1 's in column $i$ starting at the main diagonal and going down (and around) as far as possible, i.e., until a 0 is encountered. Let $V_{i}$ be the analogous set of 1 -entries in row $i$ starting at the main diagonal and going right (thus entry $(i, j)$ is in $V_{i}$ if and only if entry $(j, i)$ is in $U_{i}$ ). Then $M$ is said to have quasi-circular 1 's if the $U_{i}$ 's and $V_{i}$ 's contain all the 1's in $M$ (see Figure 1c; an augmented adjacency matrix can be arranged to have circular 1 's in each column but not quasi-circular 1's).

THEOREM 2. G is a circular-arc graph if and only if its vertices can be indexed so that $M^{*}(G)$ has quasi-circular 1 's.

Proof (Sketch). Let $S$, a family of arcs on a circle, be an intersection model for the graph $G$. Without loss of generality, we may assume that $S$ is chosen so that (a) none of its arcs equals the whole circle, (b) the arcs are closed (i.e., they contain their endpoints), and (c) no two arcs have a common counterclockwise endpoint. Starting with an arbitrary arc, index the arcs of $S$ by the order in which their counterclockwise endpoints occur as one goes clockwise around the circle (see the example in Figure 1b). Let $x_{i}$ be the vertex of $G$ corresponding to $\operatorname{arc} A_{i}$. We claim that with this indexing of the vertices, $M^{*}(G)$ now has quasi-circular 1's.

Suppose the $n$ vertices of $G$ are indexed so that $M^{*}(G)$ has quasicircular 1's. For $i=1,2, \cdots, n$, let $p_{i}$ label the $i$ th hour point on an $n$-hour clock (see example in Figure 1). Suppose $U_{i}$, the maximal circular set of 1 's starting from the main diagonal in column $i$ of $M^{*}(G)$, ends at entry $\left(m_{i}, i\right)$. Then draw $\operatorname{arc} A_{i}$ with counterclockwise endpoint $p_{i}$ and clockwise endpoint $p_{m_{i}}$ (possibly $m_{i}=i$ and $\operatorname{arc} A_{i}$ is a point; if column $i$ is all 1 's, $m_{i}=i-1$ or $m_{i}=n$ if $\left.i=1\right)$. The $A_{i}$ 's form an intersection model for $G$. Q.E.D.

Observe that the proof of Theorem 2 gives a simple algorithm for constructing a circular-arc model for $G$ when $M^{*}(G)$ has quasi-circular 1's. Unfortunately, we currently lack an algorithm to test whether $M^{*}(G)$ can have quasi-circular 1's. However, there are two important subclasses of circular-arc graphs for which we have both efficient tests (based on the Fulkerson-Gross algorithm and Theorem 1) and simple constructions of circular-arc models. These are proper circular-arc graphs and the graphs $G$ such that $M^{*}(G)$ has the circular 1 's property for columns.

TheoRem 3. $G$ is a circular-arc graph if $M^{*}(G)$ has the circular 1's property for columns.

A symmetric $(0,1)$-matrix is said to have circularly compatible 1's 
if the 1's in each column are circular and if, after inverting and/or cyclicly permuting the order of the rows and (corresponding) columns, the last 1 (in cyclicly descending order) of the circular set of 1 's in the second column is always at least as low as the last 1 of the circular set in the first column unless one of these columns is all 1's or all 0's (inversion and cyclic permutation do not affect the circularity of the 1 's).

THEOREM 4. $G$ is a proper circular-arc graph if and only if there is an arrangement of $M^{*}(G)$ with circularly compatible 1 's.

\section{REFERENCES}

1. S. Benzer, On the topology of genetic fine structure, Proc. Nat. Acad. Sci. U.S.A. 45 (1959), 1607-1620.

2. J. Cohen, Interval graphs and food webs, The RAND Corporation D-17696-PR.

3. D. R. Fulkerson and O. A. Gross, Incidence matrices and interval graphs, Pacific J. Math. 15 (1965), 835-855. MR 32 \#3881.

4. P. C. Gilmore and A. J. Hoffman, A characterization of comparability graphs and of interval graphs, Canad. J. Math. 16 (1964), 539-548. MR 31 \#87.

5. H. Hadwiger and H. Debrunner, Kombinatorische Geometrie in der Ebene, Inst. Math., Univ. Genève, Geneva, 1960; English transl., Holt, Rinehart and Winston, 1964, p. 54. MR 22 \#11310; MR $29 \# 1577$.

6. D. Kendall, Incidence matrices, interval graphs, and seriation in archeology, Pacific J. Math. 28 (1969), 565-570. MR 39 \#1344.

7. V. Klee, What are the intersection graphs of arcs in a circle?, Amer. Math. Monthly 76 (1969), 810-813.

8. C. G. Lekkerkerker and J. Ch. Boland, Representation of a finite graph by a set of intervals on the real line, Fund. Math. 51 (1962), 45-64.

9. F. S. Roberts, Representations of indifference relations, Doctoral Dissertation, Stanford University, Stanford, Calif., 1968.

10. - Indifference Graphs, Proof Techniques in Graph Theory, Proc. Second Ann Arbor Graph Theory Conf., Academic Press, New York, 1969. M3706

Mathematics Research Center, United States Army, Madison, Wisconsin 